\title{
Particle Physics for Primary Schools: Enthusing future physicists
}

\section{Cristina Lazzeroni*}

School of Physics and Astronomy, University of Birmingham, Birmingham B15 2TT, UK

E-mail: c.lazzeroni@bham.ac.uk

\section{Maria Pavlidou}

School of Physics and Astronomy, University of Birmingham, Birmingham B15 2TT, UK

E-mail: m.pavlidou@bham.ac.uk

This report summarises the work done at the University of Birmingham, UK, in collaboration with Odgen Trust Fellows to develop a set of resources for teaching basic concepts of Particle Physics in primary schools. The particle physics workshop explores the ability of young children to be imaginative and creative, and exploits it to teach them the fundamentals of particle physics in a fun way.

38th International Conference on High Energy Physics 3-10 August 2016

Chicago, USA

${ }^{*}$ Speaker. 


\section{Introduction}

In recent years, the realisation that children make decisions and choices about subjects they like in primary school, especially in UK, became widely understood. For this reason academic establishments started focussing some of their public engagement activities towards the younger ages. Besides, although particle physics is not part of the UK primary school curriculum, it ideally offers exposure to cutting edge research and fundamental discoveries, and in doing so it can inspire students towards science and effectively illustrate how science works more generally. Finally, the world of fundamental particles is a topic that shares familiar trends with concepts that children are used to, and this familiarity can be exploited to enlarge and consolidate students knowledge. Young children should not be kept away from challenging concepts because they have the ability to understand them as long as they can be related to their world.

The idea of creating a particle workshop for primary schools was born out of a collaboration between Lazzeroni, Odgen Trust Teaching fellows and local primary teachers. Taking advantage of a long-standing experience in particle physics research, the workshop for primary schools has been designed and trialled. The workshop allows young children (ages 8-11) to learn the world of fundamental particles, and use creative design and creative writing to improve and consolidate their knowledge. The workshop has already been trialled in a large number of UK primary schools and at a CERN Teachers Workshop, receiving very positive evaluation. The initial resources were reviewed and improved, based on the feedback received from school teachers and communicators; they have later been incorporated into a European project, and became also part of the educational portfolio of the UK Ogden Trust. The Birmingham Particle group has a long standing tradition of studying the quark and gluon structure of the proton in fine detail [1] and the groupÕs efforts are currently leading the top and heavy flavour physics and heavy quarkonia studies in the ATLAS experiment at the LHC [2]. Lazzeroni has studied the behaviour of strange and beauty quarks throughout her career, in several experiments including the NA48, NA62 and LHCb experiments at CERN [3].

\section{Particles: the basics}

Primary school students are generally familiar with the idea that everything around them is made out of small particles. However there is a wide variety in the depth of their knowledge: in some cases students know already the terms atom and molecule, in other cases they don't. At the start of the workshop these ideas are introduced in an interactive manner, via a discussion around the smallest thing they can think of. The terms cell or bacteria are the most commonly received answers. Students then learn that cells, microbes, bacteria etc are all made out of smaller particles which are called molecules and that these in turn are made out of even smaller particles, called atoms. Students are often familiar with the idea that it is possible to see small organisms using a microscope; it is therefore important to explain that a school microscope is not powerful enough to show atoms. The basic example of a Russian doll is pushed further, explaining that atoms are made out of smaller particles, called protons and neutrons, and these in turn are made out of even smaller particles, called quarks and gluons. No microscope can see inside an atom and students are bound to ask the obvious question: How do we know what is inside the atom if we cannot see 
it? Instead of giving a direct answer, a question can be posed here: What will you do if you want to find out how a toy works? The answer usually received is: take it apart, or smash it and see what is inside. This analogy introduces, in a very easy and simple to understand way, the reason why scientists accelerate particles and smash them together. It is important to point out that this is how scientists found the structure of the atom and the structures of protons and neutrons, therefore introducing the concept of the particle accelerator as a complex machine able to smash particles together and break them apart in order to reveal what they are made out of. The discussion can be made more lively by the use of demonstrations, like a giant-sized model of the atom that reveals the components, or the effect of scattering balls on objects. The workshop is also an opportunity to introduce CERN to the students. The location of CERN on a map of Europe and the size of the Large Hadron Collider (LHC) are discussed with the students. Aerial views of the CERN location allow to make connections with notions from European geography. The length and size of the LHC can be compared to local landmarks. The remarkable engineering achievements of LHC can be presented, for example that particles move very fast along the LHC, almost as fast as light, and complete about 10000 rounds in the blink of an eye. The detectors of the LHC can be compared to very fast digital cameras, able to take hundreds of thousands of pictures in one second, in order to capture the collisions between the fast moving particles [4].

In the next stage students are introduced to the three families of fundamental particles: the quarks, the leptons and the bosons. Soft toys, as those by the Particle Zoo [5], can be used as a visual reference to the world of fundamental particles of matter and antimatter. Students are then told various fun stories about each and every particle [6] so that they can familiarise themselves with the names but also start forming in their minds a persona for each particle. Students are also introduced to the concept of charge as a characteristic that can differ from one particle to another. In order for the students to familiarise themselves with the names of the fundamental particles and their characteristics and behaviour, students can play the Happy Families and Snap games using custom-made cards (see ??). The set of cards contains one card for each fundamental particle, with the name and family name of the particle, its mass and its charge [7]. Each card also lists some general information about the particle as well as the names of the other particles it interacts with - the concept of interaction is here simplified as liking or disliking. One card set consists of the thirty fundamental particles of matter and antimatter: six quarks (up, down, charm, strange, top, beauty), six anti-quarks (anti-up, anti-down, anti-charm, anti-strange, anti-top, anti-beauty), six leptons (electron, electron neutrino, muon, muon neutrino, tau, tau neutrino), six anti-leptons (antielectron, electron anti-neutrino, anti-muon, muon anti-neutrino, anti-tau, tau anti-neutrino) and six bosons (gluon, photon, Z, W plus, W minus, Higgs). Each card is divided into two sections, to allow both games to be played with the same set of cards. As a result of our trials, we can say that the games are a great way to embed and consolidate information. It is essential at this stage to point out to students that the cards reflect our current knowledge, that was very different years ago and probably will be very different in years to come.

The main ideas conveyed during this introductory phase of the workshop can be summarised as: particles as fundamental building blocks of matter; accelerators as prime instruments to investigate the smallest things we know of, colliding and smashing particles together; detectors as the way in which images of what happens can be captured; fundamental particles can be classified in three families, according to their characteristics and behaviour; relation between matter and antimatter; 
our scientific knowledge is evolving with time.
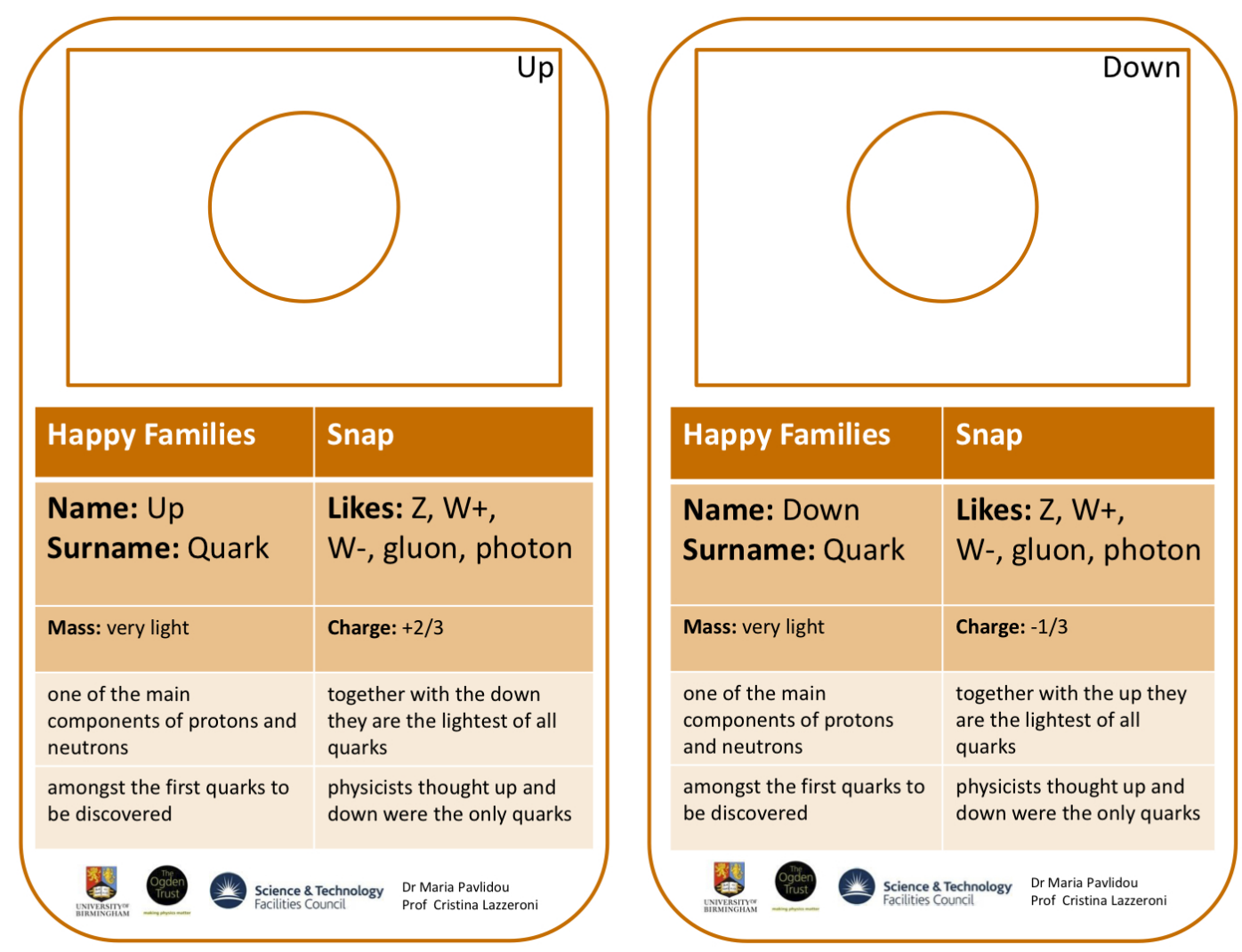

Figure 1: Example of card used in the custom-designed Happy Family and Snap games.

\section{Creativity in action: making models and writing stories of the fundamental particles}

After playing the Happy Families game students are separated into five groups of six: quarks, anti- quarks, leptons, anti-leptons and bosons. Each student is assigned one particle and is given the relevant trump card. The division is ideal for a class of thirty students. Students are asked to use their imagination and creativity to come up with a design for their particle in a way that it reflects its characteristics and properties. Students working on matterĐantimatter pairs are asked to work together because their particles have to be identical in every other way apart from the one feature which would correspond to charge. This feature can for example be coloured white for matter and black for antimatter; throughout our workshops students have come up with a variety of choices on how to represent charge by using a hat, moustache etc. Overall we found that students who worked on matterĐantimatter pairs collaborated very well, even with pupils from other schools: they were able to make suggestions, discuss their ideas and come to a common agreement. Collaborating with strangers introduces the idea that scientists work in teams, often from different countries, and that good communication and exchange of ideas is crucial in forming new scientific theories. 
When introducing fundamental particles it is important to describe mass as one of the characteristics that differentiate particles from one another, as the concept of mass relates to one of the foremost questions of current particle physics research. Masses of fundamental particles differ vastly, and makes it impossible to create a simple model where the relative masses of the particles are represented accurately. For this reason we decided to simplify and group the fundamental particles in four main categories: very light, light, heavy and very heavy. Once students have completed the design of their particle, they are given a plastic coloured ball to make the particle model. We recommend to use three different coloured sets, one for each of the families of quarks, leptons and bosons. Students are asked to fill in the plastic ball with a certain amount of plasticine in relation to them being light or heavy. Once the particles acquired mass the models were completed using a wide variety of decorations. The imagination and creativity the students showed during the completion of particle models has been impressive (see examples in figures 2 and 3 ). When evaluating the workshop students told us that this was the most enjoyable part of the day. They liked the freedom of choice they were given in making their particle model and how the characteristics of a particle can be shown in an artistic way.

The third phase of the workshop consists in a session of creative writing: starting from the newly assimilated concepts and with the help of the card games, students are challenged with writing a story about the particle which model they have produced, highlighting its characteristic and behaviour. They can use any format they like, being narrative or poetry or drama or song. This phase is one of the most enjoyable for the students and teachers, and we have witnessed the creation of truly amazing items during our workshops.

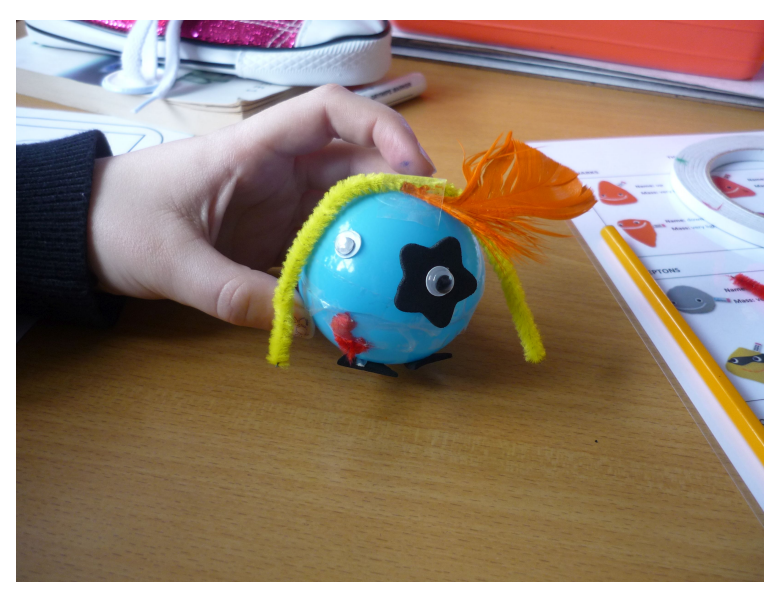

Figure 2: Detail of a particle model.

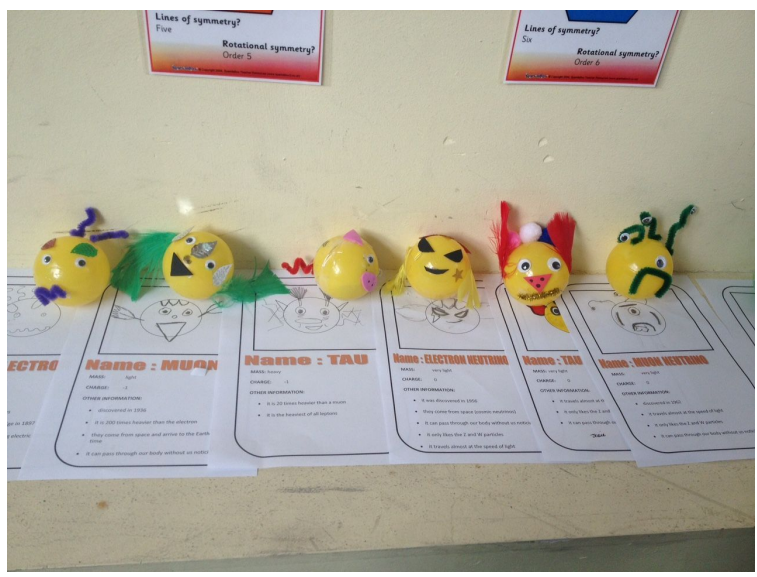

Figure 3: Classroom display with particle models.

\section{Evaluation}

During our workshops we evaluated the engaging strength of the activities, the enjoyment the students demonstrated and the short term learning of the students. Students were able to successfully answer questions such as: Where is CERN?, What does the LHC do?, How do scientists find out about the structure of small things such as atoms?, How many particle families do we have and what are their names?, How many quarks / leptons/bosons can you name?, Do you remember any 
special features of a particle?. The short term learning was very high, with a class of typically 30 students being able to answer all these questions easily.

In order to evaluate if students understood the idea that science is progressing and that what we know evolves with time we also asked the question: Can you tell us something about how science works?. The student comments included: we might find in a few years that there are smaller particles inside quarks; maybe there are more particles we have not discovered yet; and finally, I will become a particle physicist to find new particles. These comments made it clear that the workshop fulfilled its aims: to teach, to enthuse and to give a wider understanding of how science works.

Looking ahead, we plan to evaluate the long term learning of the students. Our next step is to offer training to school teachers, so that they can use this workshop in their classroom as and when they choose and therefore have the means to complete a long-term evaluation on the learning of the students. We hope that this workshop will provide inspiration for teachers and will be used as a cross-curricular project within schools.

\section{Conclusions and Acknowledgments}

The resources we created are based on particle physics research and form an enjoyable and successful way to engage with a primary school audience, while inspiring and enthusing students to pursue their interest in science. The workshop has been delivered in primary schools in UK and forms an excellent framework for a collaboration between academics, local teachers and educational officers that push the boundaries of traditional public engagement, extending it to a lower age audience and achieving impact trough the quality of the interaction.

We would like to thank Amanda Poole, Ogden School Teacher Fellow, for her initial request to make a particle physics workshop for primary schools and for allowing us to trial it first with her students. We would like to thank STFC and The Ogden Trust for their support in establishing and running this workshop. We would finally like to thank Phill Day of STFC for his very useful input and guidance on reviewing the resources of the Particle Physics Workshop.

\section{References}

[1] H1 experiment at HERA (http://h1.desy.de)

[2] ATLAS experiment public results (https://twiki. cern.ch/twiki/bin/view/AtlasPublic)

[3] NA48, NA62, LHCb public results (http://na48.web.cern.ch/ NA48/Welcome.html, http://na62.web.cern.ch/NA62, http://lhcb.web.cern.ch/lhcb/)

[4] G.J. Wiener, J. Woithe, A. Brown and K. Jende, Introducing the LHC in the classroom: an overview of education resources available, Phys. Educ. 51035001 (2016)

[5] The Particle Zoo, LLC 2015 http://particlezoo. net/

[6] A. Alexopoulos, Playing with protons in primary, CERN Document Server (http://cds.cern.ch/ record/1998688)

[7] International Particle Physics Outreach Group 2013 Quark poker (http://ippog.web.cern.ch/ resources/2012/quark-poker) 